\title{
DSG2 expression is correlated with poor prognosis and promotes early-stage cervical cancer
}

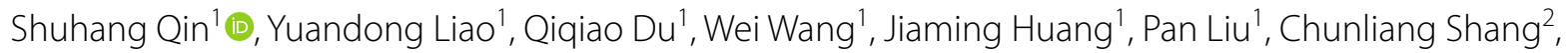
Tianyu Liu ${ }^{1}$, Meng Xia ${ }^{1}$ and Shuzhong Yao ${ }^{1 *}$ (D)

\begin{abstract}
Background: The pathogenesis and developmental mechanism of early-stage (FIGO 2009 IA2-IIA2) cervical cancer (CC) remain unclear. Seeking novel molecular biomarkers based on The Cancer Genome Atlas (TCGA) will facilitate the understanding of CC pathogenesis and help evaluate early-stage CC prognosis.

Methods: To identify prognosis-related genes in early-stage CC, we analyzed TCGA mRNA-seq data and clinical data by univariate Cox and Kaplan-Meier plotter analyses. Differential expression analysis identified upregulated genes in early-stage CC. Combined with the genes correlated with unfavorable prognosis, we selected desmoglein-2 (DSG2) for further investigation. To detect DSG2 expression in early-stage CC, we used immunohistochemistry (IHC), quantitative real-time PCR (qRT-PCR) and western blotting. The relationship between the expression of DSG2 and clinical features was analyzed by the Chi square test. Cox analysis was applied to assess the relationship between CC overall survival (OS) and risk factors. The correlations between DSG2 expression and CC cell line proliferation and migration were investigated with Cell Counting Kit-8 (CCK-8) and migration assays.

Results: There were 416 prognosis-related genes in early-stage CC. DSG2, matrix metallopeptidase 1 (MMP1), carbonic anhydrase IX (CA9), homeobox A1 (HOXA1), and serine protease inhibitor B3 (SERPINB3) were upregulated in early-stage CC compared with adjacent noncancerous tissue (ANT) and correlated with unfavorable prognosis. Among them, DSG2 was most significantly correlated with patient survival. Coexpression analysis indicated that DSG2 was probably involved in cell division, positive regulation of transferase activity, positive regulation of cell migration, EGFR upregulation pathway and regulation of lymphangiogenesis. IHC, QRT-PCR and western blotting showed that DSG2 expression was higher in CC than in normal tissue. Significant correlations were identified between DSG2 expression and several aggressive clinical features, including pelvic lymph node metastasis (PLNM). Multivariate Cox analysis showed that DSG2 and PLNM were independent prognostic factors for OS. DSG2 knockdown inhibited CC cell proliferation and migration.
\end{abstract}

Conclusions: DSG2 is a biomarker that promotes tumor proliferation and metastasis and is correlated with poor prognosis in early-stage CC.

Keywords: Desmoglein-2, Early-stage cervical cancer, Prognosis, Pelvic lymph node metastasis

\footnotetext{
*Correspondence: yszlfy@163.com

${ }^{1}$ Department of Obstetrics and Gynecology, The First Affiliated Hospital, Sun Yat-sen University, Zhongshan Second Road 58, Guangzhou 510080, People's Republic of China

Full list of author information is available at the end of the article
}

\section{Background}

Uterine cervical cancer (CC) is one of most common cancers. According to the Global Cancer Statistics 2018 report, the incidence rate and mortality of $\mathrm{CC}$ ranked

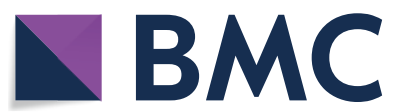

(c) The Author(s) 2020. This article is licensed under a Creative Commons Attribution 4.0 International License, which permits use, sharing, adaptation, distribution and reproduction in any medium or format, as long as you give appropriate credit to the original author(s) and the source, provide a link to the Creative Commons licence, and indicate if changes were made. The images or other third party material in this article are included in the article's Creative Commons licence, unless indicated otherwise in a credit line to the material. If material is not included in the article's Creative Commons licence and your intended use is not permitted by statutory regulation or exceeds the permitted use, you will need to obtain permission directly from the copyright holder. To view a copy of this licence, visit http://creativeco mmons.org/licenses/by/4.0/. The Creative Commons Public Domain Dedication waiver (http://creativecommons.org/publicdomain/ zero/1.0/) applies to the data made available in this article, unless otherwise stated in a credit line to the data. 
fourth among all female cancers [1]. In contrast to latestage CC patients, most early-stage (IA2-IIA2) CC patients have a significantly increased survival time after surgery and chemoradiotherapy. However, approximately $10-30 \%$ of early-stage patients were found to have pelvic lymph node metastasis (PLNM), and some of the patients eventually experienced adverse outcomes [2]. In earlystage CC, patients with moderately high-risk factors, including large tumor size $(>2 \mathrm{~cm})$, poor differentiation, special pathologic types, deep stromal invasion, lymphovascular space invasion (LVSI), PLNM and parametrial infiltration, usually have relatively shorter survival times $[2,3]$.

Currently, the pathogenesis and mechanism of CC metastasis remain unclear and probably involve the aberrant expression of numerous oncogenes and tumor suppressors. Rapid advances in molecular biotechnology revealed that some molecular biomarkers are related to the progression of CC [4]. Seeking novel molecular biomarkers of protein-coding genes would facilitate the understanding of CC pathogenesis and help us evaluate the prognosis of early-stage CC.

The Cancer Genome Atlas (TCGA) database has been developed in recent years. It is composed of a large amount of cancer mRNA-seq data as well as detailed clinical data, which makes bioinformatic data mining convenient and reliable [5]. We incorporated gene profiling, molecular signatures, and functional and pathway information with gene set enrichment analysis. Using bioinformatics analyses, we found a series of early-stage $\mathrm{CC}$ prognosis-related genes. Among all these genes, we found that desmoglein-2 (DSG2) was upregulated in early CC compared with normal samples and also predicted unfavorable prognosis in early CC.

DSG2 is a cell adhesion protein of the cadherin superfamily that is crucial for cardiomyocyte cohesion and function [6]. Its purpose is to regulate cell-cell contact with adjacent cells. The altered expression and function of desmosomal cadherins is associated with human tumorigenesis [7]. Brennan et al. [8] and Kurzen et al. [9] showed that DSG2 was more highly expressed in skin squamous cell carcinoma and basal cell carcinoma and that the positive rate was higher in high-risk patients. Kamekura et al. [10] showed that the downregulation of DSG2 inhibited the proliferation of colon cancer cells. Saaber et al. [11] showed that DSG2 was a novel biomarker of squamous cell lung carcinoma. Cai et al. [12] showed that DSG2 was more highly expressed in nonsmall cell lung cancer (NSCLC) and that the knockdown of DSG2 inhibited the progression of NSCLC. However, some studies have shown that DSG2 is expressed at lower levels in cancer and functions as a tumor suppressor. Yashiro et al. [13] showed that the high expression of DSG2 was correlated with a longer survival time among diffuse infiltrative carcinomas of the stomach. Ramani et al. [14] showed that the knockdown of DSG2 decreased the cell junction of pancreatic carcinoma cells and increased the rate of metastasis. Barber et al. [15] showed that the low expression of DSG2 was an independent prognostic factor for prostate cancer. Davies et al. [16] showed that lower-expressed DSG2 was correlated with poor differentiation, larger tumor size and lymph node metastasis in breast cancer.

However, the role of DSG2 in CC has never been explored. In the present study, we identified DSG2 as a novel $C C$ prognosis-related gene using data mining. With clinical and cell line validation, we demonstrated that it probably increased the risk of PLNM and resulted in an unfavorable prognosis.

\section{Methods \\ Datasets}

The gene expression and clinicopathological data of 310 $\mathrm{CC}$ patients and 3 adjacent noncancerous tissues (ANTs) were downloaded from TCGA (https://portal.gdc.cance r.gov/) $[17,18]$. According to the TCGA publication guidelines (https://www.cancer.gov/about-nci/organizati on/ccg/research/structural-genomics/tcga), these mRNA sequencing data have no restrictions on publication, and no additional approval by an ethics committee was required to publish the use of the data.

With the Ensembl platform (http://www.ensembl.org/), we separated the mRNAs from all the TCGA genes. Genes that had missing values in over $50 \%$ of the samples were removed. Finally, there were 12,084 genes included in the study. Samples without data on the survival state and survival time were also removed. Finally, $291 \mathrm{CC}$ tissues, including 167 early-stage (FIGO 2009 IA2-IIA2) CC tissues and 3 ANTs, were included in the study. For the early-stage samples, any missing data on whether LVSI and corpus involvement occurred were all recorded as nonoccurrence (median of the available data).

Four CC datasets from Oncomine (version 4.5) (https ://www.oncomine.org/) [19] were used to validate the results obtained from TCGA.

\section{Kaplan-Meier (KM), univariate Cox, Gene Ontology (GO), Kyoto Encyclopedia of Genes and Genomes (KEGG) and protein-protein interaction (PPI) analyses}

The prognostic value of each gene was calculated in the KM analyses and univariate Cox analyses for the earlystage cohort. A total of 416 genes with both $P_{K M}<0.05$ and $P_{C o x}<0.05$ were early-stage prognosis-related genes and were kept for further analyses. GO biological process, cellular component, and molecular function categories and KEGG pathway analyses and PPI network 
construction were conducted by the Metascape website (http://metascape.org/gp/index.html), using false discovery rate (FDR) q-value $<0.05$ as the standard for statistical significance.

\section{Differential expression analyses (DEA)}

To identify genes that are more highly expressed in early-stage CC than in ANTs, we performed a DEA of prognosis-related genes between 167 early-stage CC patients and 3 ANTs with the R package "DEseq 2". The differentially expressed mRNAs with $\log _{2}|\mathrm{FC}|>1.5$ and $P$-adjusted $<0.05$ were considered to be significant. Hierarchical clustering analysis was applied to categorize the data into two groups with similar expression patterns between early-stage CC and ANTs.

\section{Coexpression analyses}

Coexpression analyses was conducted by the cBioPortal website (https://www.cbioportal.org/). Using Spearman's correlation analyses, the genes with FDR q-value $<0.05$ were regarded as coexpressed with DSG2. Then, GO biological process analysis and oncogenic signature analysis were conducted among the positively correlated genes (Spearman's correlation $>0$ ) and negatively correlated genes (Spearman's correlation $<0$ ) by the Metascape website.

\section{Tissue sample collection}

A total of 150 CC tissues, 6 ANTs and 30 normal cervical tissues (NCTs) collected from January 2006 to October 2012 were obtained from the archives of the Pathology Department and Gynecology Department of the First Affiliated Hospital of Sun Yat-sen University. All enrolled CC patients were matched from stage IA2 to IIA2 and underwent radical hysterectomy and lymphadenectomy. Only patients with no preoperative radiotherapy or chemotherapy and with available clinical follow-up data were enrolled. Thirty NCTs were collected from patients who underwent hysterectomy without malignant conditions. Written informed consent was obtained from each patient. All specimens were handled according to legal and ethical standards.

\section{Cell lines and cell culture}

In this study, SiHa, HeLa, C33A, CaSki, MS751 and ME180 cells were purchased from the American Type Culture Collection (ATCC, Rockville, MD, USA) and cultured according to their guidelines in a humidified atmosphere with $5 \% \mathrm{CO}_{2}$ at $37{ }^{\circ} \mathrm{C}$. The $\mathrm{SiHa}, \mathrm{HeLa}$ and ME180 cell lines were cultured in DMEM (Thermo Fisher, America). The CaSki cell line was cultured in RPMI 1640 medium (Thermo Fisher, America). The C33A and MS751 cell lines were cultured in Eagle's minimum essential medium (Thermo Fisher, America). The media were supplemented with $10 \%$ fetal bovine serum (Life Technology, America) and 1\% antibiotics (100 U/ml penicillin and $100 \mu \mathrm{g} / \mathrm{ml}$ streptomycin) (Life Technology, America).

\section{Immunohistochemistry (IHC)}

For IHC, 4- $\mu$ m paraffin-embedded sections were baked at $60{ }^{\circ} \mathrm{C}$ for $1 \mathrm{~h}$, deparaffinized with xylene, rehydrated with a series of graded alcohols, and microwaved in EDTA antigen retrieval buffer. Then, the sections were blocked with $10 \%$ goat serum before incubation with a primary antibody at $4{ }^{\circ} \mathrm{C}$ overnight, followed by HRP-conjugated secondary antibody incubation for $30 \mathrm{~min}$ at room temperature. DAB was added to detect antibody binding. Once brown color appeared, the sections were immersed in distilled water to stop the reaction. The sections were counterstained with hematoxylin, dehydrated in graded alcohols and mounted. The primary antibodies were rabbit anti-human DSG2 monoclonal antibody (ab150372, Abcam, Britain) and mouse anti-human D2-40 monoclonal antibody (MAB-0567, MXB, China). The DSG2 staining results were scored based on the following criteria: (i) percentage of positive tumor cells in the tumor tissue: 0 (0\%), 1 (1-10\%), 2 (11-50\%), $3(51-70 \%)$ and 4 (71-100\%); and (ii) staining intensity: 0 (none), 1 (weak), 2 (moderate), and 3 (strong). The staining index was calculated as the staining intensity score $\times$ the proportion of positive tumor cells (range from 0 to 12). The staining score of 6 was defined as the cutoff. Thus, patients with different positive staining levels of DSG2 expression were divided into low- and high-staining groups.

\section{RNA extraction and quantitative real-time PCR (qRT-PCR)}

Total RNA was extracted using Trizol reagent (TAKARA, Japan) according to the manufacturer's instructions, and the concentration of the RNA extracts of each sample was measured quantitatively by a NanoDrop ND-2000 spectrophotometer. RNA was reverse transcribed into cDNA by using PrimeScript RT Master Mix (TAKARA, Japan). cDNA was amplified and quantified using a 7500 Fast Real-Time PCR system (Applied Biosystems, USA) and SYBR Premix Ex Taq (TAKARA, Japan). The RTPCR conditions for genes were set at $95^{\circ} \mathrm{C}$ for $2 \mathrm{~min}$, followed by 39 cycles at $95{ }^{\circ} \mathrm{C}$ for $20 \mathrm{~s}, 58^{\circ} \mathrm{C}$ for $30 \mathrm{~s}$ and $72{ }^{\circ} \mathrm{C}$ for $30 \mathrm{~s}$. The DSG2 sequences were $5^{\prime}$-CTCAGG TGTGCAGCCTACTC-3' (forward) and 5'-GTGGTG TTCCTAGCCGTCAT- $3^{\prime}$ (reverse), while the GAPDH sequences were $5^{\prime}$-TGCACCACCAACTGCTTAGC-3' (forward) and $5^{\prime}$-GGCATGGACTGTGGTCATGAG$3^{\prime}$ (reverse). qRT-PCR was repeated at least three times. mRNA expression was defined based on $\mathrm{Ct}$, and relative expression levels were calculated using the comparative 
Ct $\left(2^{-\Delta \Delta C t}\right)$ method after normalization with reference to the expression of the house-keeping gene GAPDH.

\section{Western blot assay}

Total protein was extracted with cold RIPA lysis buffer and fractionated by sodium dodecyl sulfate-polyacrylamide gel electrophoresis (SDS-PAGE) and then transferred onto a $0.45-\mu \mathrm{m}$ PVDF membrane (Millipore, America). The membranes were blocked with 5\% skimmed milk and incubated with the primary antibody at $4{ }^{\circ} \mathrm{C}$ overnight, followed by secondary antibody incubation for $1 \mathrm{~h}$ at room temperature. Bound antibodies were detected with Immobilon Western Chemiluminescent HRP Substrate (Millipore, America). Rabbit antihuman DSG2 monoclonal antibody (ab150372, Abcam, Britain) and rabbit anti-human GAPDH antibody (XS20180808002, Bioworld, China) were used in this study.

\section{siRNA-mediated knockdown of DSG2}

$\mathrm{SiHa}$ and HeLa cells were transfected with control siRNA (GenePharma, Shanghai, China) or DSG2-specific siRNA (GenePharma, China) using Lipofectamine RNAiMAX Reagent (Invitrogen, America) and Opti-MEM media (Life Technology, America) at the time of cell culture. There were two DSG2 siRNA sequences. The siRNA393 sequences were 5'-CCAAUUGCCAAGAUACAUUTT- ${ }^{\prime}$ (forward) and 5'-AAUGUAUCUUGGCAAUUGGTT-3' (reverse). The siRNA613 sequences were $5^{\prime}$-CCUUAG AGCUACGCAUUAATT-3' (forward) and 5'-UUAAUG CGUAGCUCUAAGGTT- $3^{\prime}$ (reverse). The negative control sequence (siRNA-NC) was 5'-UUCUCCGAACGU GUCACGUTT-3' (forward) and 5'-ACGUGACACGUU CGGAGAATT-3' (reverse).

\section{Cell Counting Kit-8 (CCK-8) assay}

For the CCK- 8 assay, $5 \times 10^{3} \mathrm{SiHa}$ and HeLa cells were seeded into each well of 96 -well plates. The time calculation started when the cells adhered to the wall, and the wells were transfected with siRNA. Cell viability was measured at specific times by CCK-8 (CCK-8, DOJINDO, Japan). The absorbance value at $450 \mathrm{~nm}$ was read by a microplate reader (Tecan Sunrise, Tecan Group Ltd.).

\section{Migration assay}

The stable cell lines SiHa siRNA-NC, SiHa siRNA393, SiHa siRNA613, HeLa siRNA-NC, HeLa siRNA393 and HeLa siRNA613 were counted and then $10 \times 10^{4}$ stably infected SiHa cells and $20 \times 10^{4}$ stably infected HeLa cells in $250 \mu \mathrm{l}$ of serum-free medium were separately plated into the upper chamber of $8-\mu \mathrm{m}$ transwell inserts (BD Biosciences, Franklin Lakes, NJ), while $500 \mu \mathrm{l}$ of medium containing $10 \%$ bovine serum albumin was added to the lower chamber. After $24 \mathrm{~h}$ of incubation at $37^{\circ} \mathrm{C}$, SiHa siRNA cells in the upper chamber were removed carefully. After $48 \mathrm{~h}$ of incubation at $37{ }^{\circ} \mathrm{C}$, HeLa siRNA$\mathrm{NC}$ and HeLa siRNA cells in the upper chamber were removed. Migrated cells on the lower membrane surface were fixed in $4 \%$ paraformaldehyde (Solarbio, Beijing, China) for $10 \mathrm{~min}$ and then stained with $0.1 \%$ crystal violet (KeyGEN biotech, Nanjing, China) for $10 \mathrm{~min}$. The number of cells was counted in 5 randomly selected visual fields $(100 \times)$ per well under an inverted microscope DMI4000B (Leica, Wetzlar, Germany).

\section{Statistical analyses}

Statistical analyses were performed using SPSS 22.0 statistical software (Chicago, IL, USA) and R version 3.6.0. The differences between two groups were analyzed by Student's $\mathrm{t}$ test. The differences among more than two groups were analyzed by ANOVA. The Chi square test and Fisher's exact test were used to analyze the relationship between DSG2 expression and the clinicopathological characteristics. Survival data were evaluated using univariate and multivariate Cox regression analyses. Survival curves were plotted by the KM method and compared using the log-rank test. In all cases, $P<0.05$ was considered statistically significant.

\section{Results}

Early-stage CC prognosis-related genes were identified by bioinformatic analyses

According to the KM plotter analyses and univariate Cox analyses, the TCGA data included 416 early-stage prognosis-related genes, including 217 protective (Cox coefficient $<0$ ) and 199 hazardous (Cox coefficient $>0$ ) genes. In the $\mathrm{GO}$ analyses using all survival-related genes, 4 biological process terms were significantly enriched ( $P$-adjusted $<0.05)$, including the regulation of the mitotic cell cycle, the nucleobase-containing small molecule metabolic process, protein N-linked glycosylation, and organelle localization. GO cellular component analyses identified endoplasmic reticulum lumen as the significantly enriched signature (P-adjusted $<0.05)$. No signatures were significantly enriched in GO molecular function analysis. KEGG pathway analyses indicated that the significant pathways were purine metabolism, protein processing in the endoplasmic reticulum, and nucleotide excision repair (Fig. 1b, Additional file 1: Figure S1a). Each chromosome had different numbers of up- and downregulated prognosis-related genes (Additional file 1: Figure S1b). Additionally, we constructed a PPI network to interpret the potential biological roles of the prognosis-related mRNAs in early-stage CC (Additional file 1: Figure S1c). 


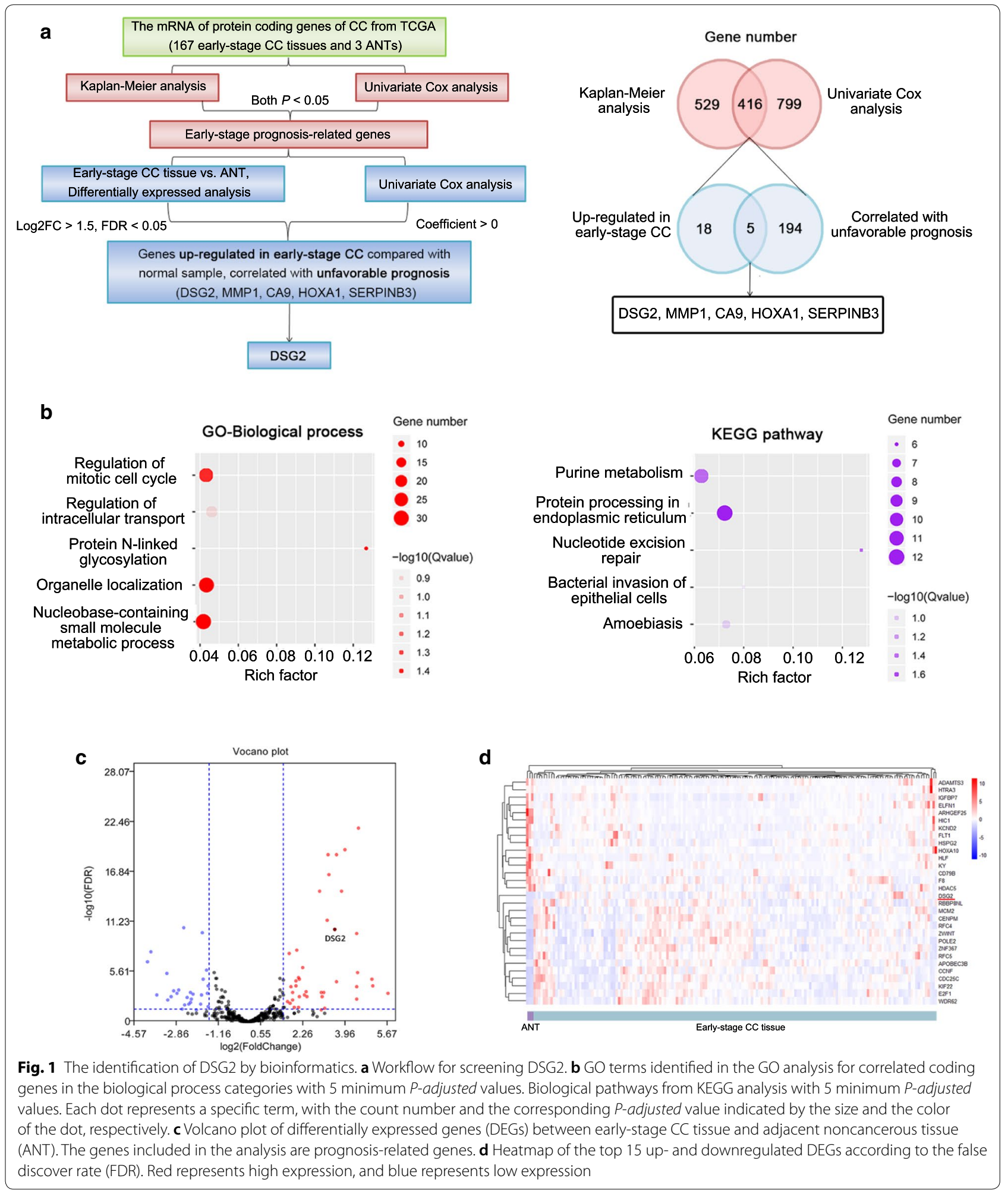

\section{DSG2 was identified by bioinformatic analyses}

DEA of prognosis-related genes identified 24 upregulated genes and 171 downregulated genes in CC compared with ANTs. The volcano plot and heatmap of the differentially expressed genes (DEGs) are shown in Fig. 1c, d. DSG2 was included in the top 15 upregulated genes. 
Five overlapping genes between hazardous (Cox coefficient $>0, P_{K M}<0.05$ and $\left.P_{C o x}<0.05\right)$ genes and upregulated genes $\left(\log _{2} \mathrm{FC}>1.5, \mathrm{FDR}<0.05\right)$ in $\mathrm{CC}$ were identified. Except for DSG2, the other four genes have been explored in CC. Therefore, DSG2 was used for further validation in clinical samples and cells. The workflow for screening DSG2 and the 5 overlapping genes were shown in Fig. 1a and Table 1.

\section{The potential functions of DSG2 in CC and other cancers were analyzed by bioinformatics}

For further validation, we investigated the difference in DSG2 expression between normal tissue and CC based on Oncomine datasets (Fig. 2a). All datasets revealed that DSG2 was upregulated in the cancer group. Survival analyses of both the overall cohort and early-stage cohort showed that the expression of DSG2 predicted an unfavorable prognosis in $\mathrm{CC}$ (overall cohort: $\mathrm{HR}=1.966$, $P=0.006$; early-stage cohort: $\mathrm{HR}=2.122, \quad P=0.030$ ) (Fig. 2c). Furthermore, the overall cohort survival analyses showed that the expression of DSG2 predicted an unfavorable prognosis in bladder urothelial carcinoma (BLCA), brain lower-grade glioma (LGG), lung adenocarcinoma (LUAD), pancreatic adenocarcinoma (PAAD) and uterine corpus endometrial carcinoma (UCEC), while predicting a favorable prognosis in colon adenocarcinoma (COAD), kidney renal clear cell carcinoma (KIRC) and kidney renal papillary cell carcinoma (KIRP) (all $P<0.05$ ) (Fig. 2b).

Genes that were coexpressed in conjunction with DSG2 were identified with cBioPortal analyses

\begin{tabular}{ll}
$\begin{array}{l}\text { Table } 1 \text { The up-regulated genes in early-stage CC } \\
\text { tissue compared with ANT, correlated with unfavorable } \\
\text { prognosis }\end{array}$ \\
\hline Gene & Description \\
\hline DSG2 & Desmoglein-2 \\
MMP1 & Matrix metallopeptidase 1 \\
CA9 & Carbonic anhydrase IX \\
HOXA1 & Homeobox A1 \\
SERPINB3 & Serine protease inhibitor B3 \\
\hline
\end{tabular}

(P-adjusted $<0.05)$. There were 2610 positively correlated genes (Spearman's correlation $>0$ ) and 2737 negatively correlated genes (Spearman's correlation $<0$ ). The enriched GO biological process and oncogenic pathway items are shown in Fig. 2e and Additional file 1: Figure S1d. According to the positively correlated gene enrichment, cell division, positive regulation of transferase activity, positive regulation of cell migration and the EGFR upregulation pathway were significantly enriched (P-adjusted $<0.05)$, revealing that DSG2 is involved in the process and metastasis of CC. Furthermore, two genes were significantly coexpressed with DSG2, CCBE1 and VASH1, which are genes that regulate lymphangiogenesis according to GO biological process analyses (Fig. 2d). DSG2 was positively correlated with CCBE1, which positively regulated lymphangiogenesis, while it was negatively correlated with VASH1, which negatively regulated lymphangiogenesis.

These results confirmed that DSG2 was important in the development of various cancers and was possibly an oncogenic gene in CC.

\section{DSG2 expression is upregulated in CC tissues}

IHC was performed on 150 early-stage CC samples and 30 NCTs, and the results revealed that DSG2 was more highly expressed in CC samples (Fig. 3b). Furthermore, DSG2 expression was upregulated in six early-stage CC samples compared to that in matched ANTs derived from the same patients (Fig. 3a).

Additionally, to validate the results above, the DSG2 mRNA expression level was determined in 20 NCTs and 20 early-stage CC tissues using qRT-PCR. Moreover, $3 \mathrm{NCT}$ and 3 early-stage CC tissues were randomly selected from the abovementioned tissues for western blot analyses. A comparison of the results showed that DSG2 mRNA and protein levels were higher in earlystage CC tissues than in NCTs (Fig. 3c).

\section{High expression of DSG2 is associated with poor clinical features and prognosis in early-stage CC \\ The correlation between DSG2 expression and clinico- pathological features was analyzed according to the IHC score. High DSG2 expression was significantly correlated with several poor clinicopathological features, including}

\footnotetext{
(See figure on next page.)

Fig. 2 The potential functions of DSG2 in CC and other cancers. a Box plots of DSG2 expression based on Oncomine datasets. DSG2 was significantly overexpressed in cancer in the Scotto Cervix 2, Zhai Cervix, Pyeon Multi-cancer and Biewenga Cervix datasets. b Kaplan-Meier survival curves of DSG2 showing the overall survival outcomes by relatively high DSG2 expression and low DSG2 expression in patients with cancers except CC. c Kaplan-Meier survival curves of DSG2 showing the overall survival outcomes in the overall cohort and early-stage cohort by relatively high DSG2 expression and low DSG2 expression in CC patients. $\mathbf{d}$ The correlation between DSG2 and two genes regulating lymphangiogenesis. e GO terms identified in the GO biological process analysis for positively coexpressed genes in categories with 20 minimum $P$-adjusted values. Oncogenic signature for positively coexpressed genes with 20 minimum P-adjusted values
} 
a Scotto Cervix 2

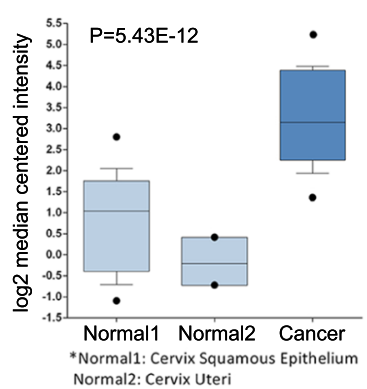

b

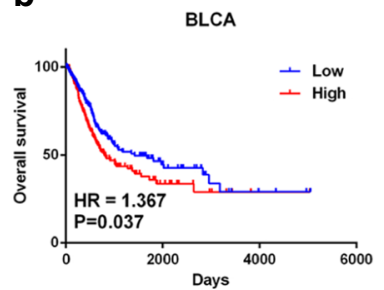

LGG

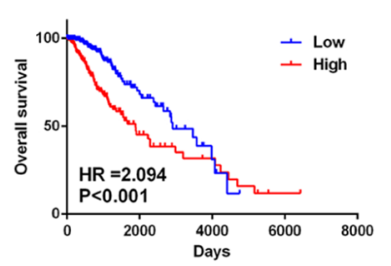

C
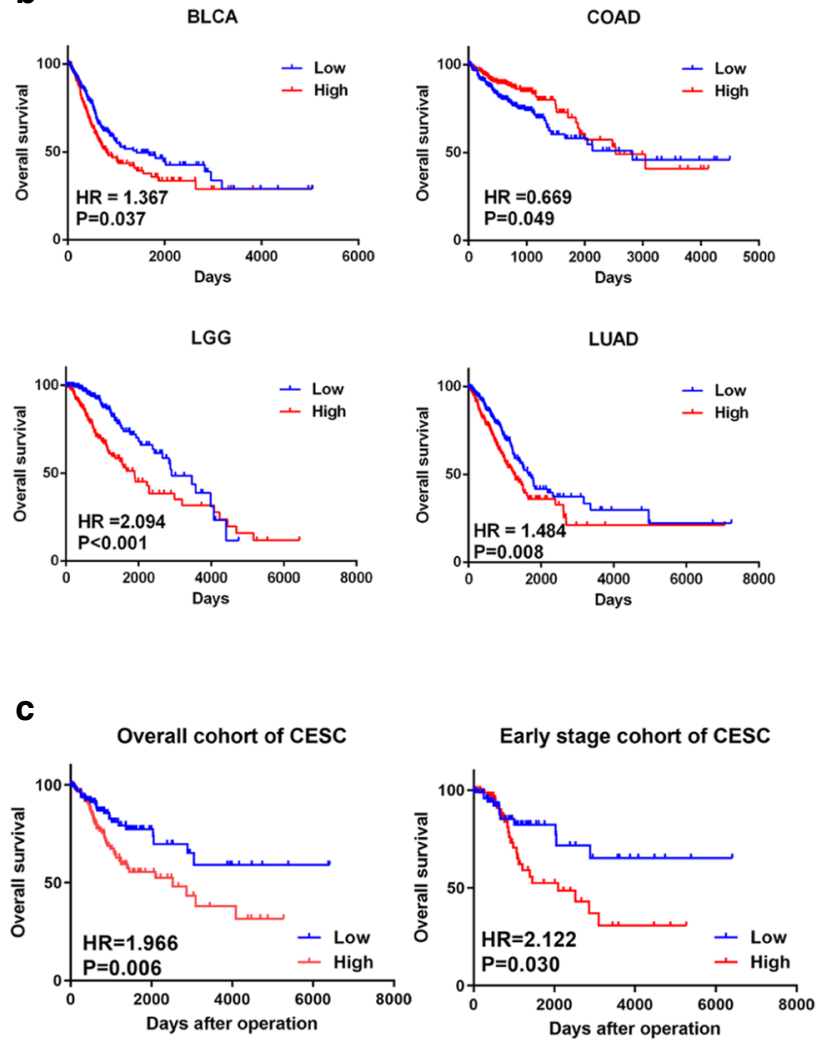

LUAD

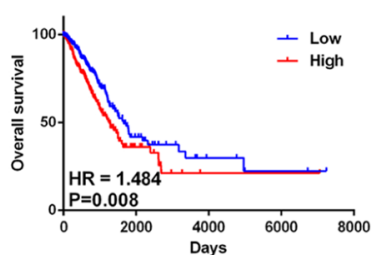

d

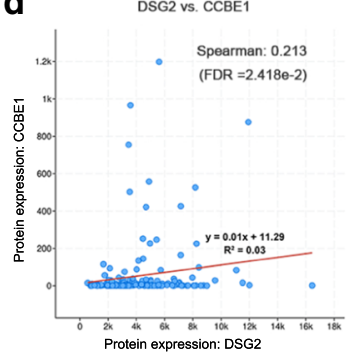

Pyeon Multi-cancer

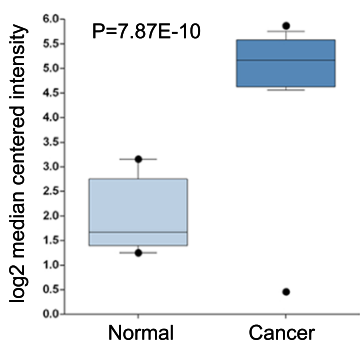

KIRC

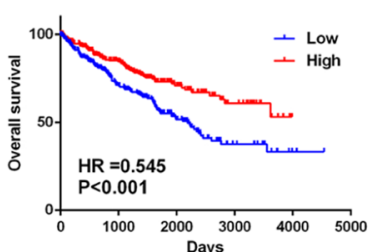

PAAD
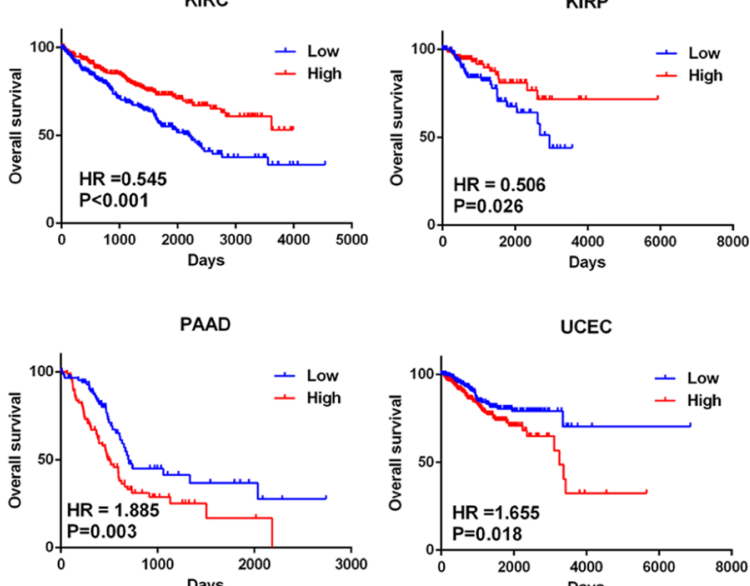

UCEC
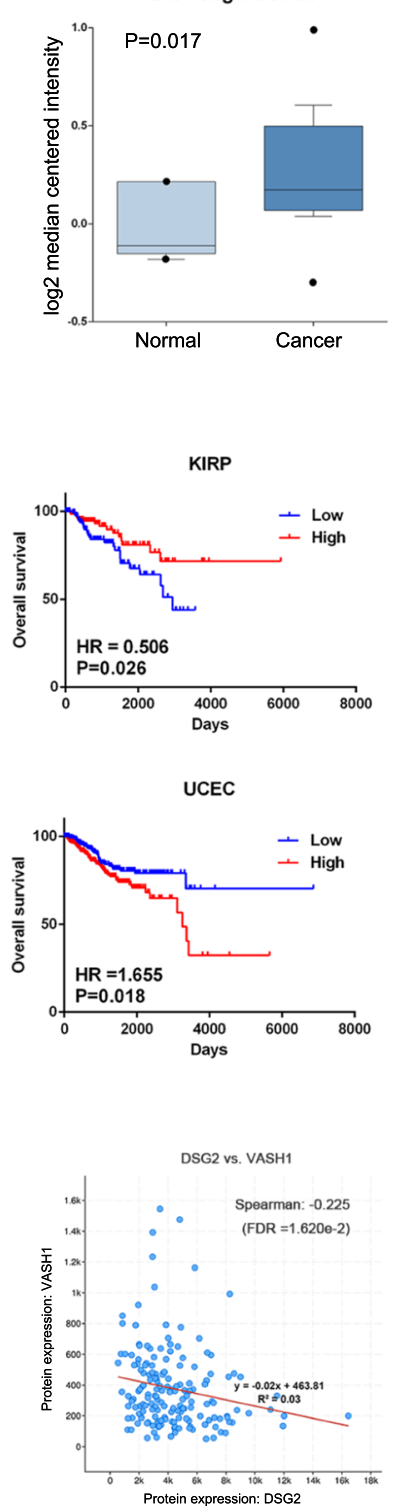

e Positive correlated genes

GO-Biological process

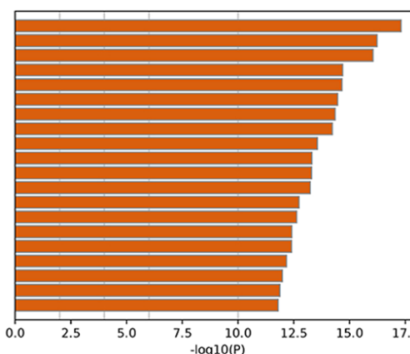

Oncogenic signature 60:0080135: regulation of cellular response to stress
G0:0007169: transmembrane receptor protein tyrosine kinase signaling pathway 0:0000904: Cell morphogenesis involved in differentiat
60:0051301: cell division 60:0051347: positivis requlation of transferase activity 60:0007017: microtubuli-ebased process :0034330: cell junction organization G0:0043087: regulation of GTPase activity :0048732: gland development 0.0048589 : developmente catabolic process 60:0045786: negative regulation of cell cycle 60:0030036: actin cytoskeleton organization 0:1903827: regulation of cellular protein localization 60:0016569: covalent chromatin modification 0:0030335: positive regulation of cell migration 


\section{(See figure on next page.)}

Fig. 3 The expression of DSG2 in patient samples was determined by IHC, qRT-PCR and western blotting. a IHC was performed on six pairs of matched CC samples and adjacent nontumor cervical tissue (ANT) samples. Original magnifications: $\times 200$. b Representative results of the IHC of DSG2 in normal samples and early-stage CC samples with and without PLNM. Bar graph shows the percentage of high/low expression of DSG2 in normal samples and early-stage cohort samples. cThe mRNA expression of DSG2 was determined by qRT-PCR in 20 NCTs and 20 CC samples (left). The protein expression of DSG2 was determined by western blotting in 3 NCTs and 3 CC samples (right). $\mathbf{d}$ The mRNA expression of DSG2 was determined by qRT-PCR in 20 CC samples without PLNM and 20 cervical cancer samples with PLNM (left). The protein expression of DSG2 was determined by western blotting in 3 CC samples without PLNM and 3 CC samples with PLNM (right). e Kaplan-Meier curve for DSG2 and PLNM in early-stage cohort CC patients. DSG2 (-): low expression of DSG2; DSG2 (+): high expression of DSG2. PLNM (-): without PLNM; PLNM (+): with PLNM. f Representative results of the IHC of lymphatic vessel density in CC with high/low expression of DSG2. Original magnifications: 100x

tumor size $(P=0.022)$, PLNM $(P<0.001)$, recurrence $(P<0.001)$ and vital status in 5 years $(P<0.001)$ (Table 2$)$. No significant correlation was identified between DSG2 expression and age, FIGO stage, pathologic type, differentiation grade, stromal invasion, LVSI, vaginal involvement and parametrial infiltration (Table 2). To verify the relationship between DSG2 expression and the prognosis of early-stage CC, univariate and multivariate Cox analyses were performed. Univariate analysis showed that DSG2 expression $(P<0.001)$, tumor size $(P=0.029)$, LVSI $(P=0.008)$ and PLNM $(P<0.001)$ were prognostic factors for overall survival (OS) (Table 3). Multivariate analysis showed that DSG2 expression $(P=0.018)$ and PLNM $(P=0.006)$ were independent prognostic factors for OS (Table 4, Fig. 3e).

\section{High DSG2 expression was correlated with the occurrence of PLNM}

The IHC results showed that DSG2 expression was significantly correlated with PLNM (Table 2, Fig. 3b). For further validation, qRT-PCR was performed to examine the mRNA levels of DSG2 in 20 PLNM and 20 nonPLNM tissues, while western blotting was performed to examine the protein levels of DSG2 in 3 PLNM and 3 non-PLNM tissues. Both the mRNA and protein levels of DSG2 in the PLNM group were higher than those in the non-PLNM group $(P<0.05)$ (Fig. $3 \mathrm{~d})$. This validation result was consistent with the IHC result.

Moreover, to explore the mechanism of how DSG2 promoted PLNM, we detected the lymphatic microvessel density (LMVD) in the same IHC samples. We found that the high DSG2 expression group had higher LMVD than the low DSG2 group, indicating that DSG2 probably promoted PLNM by promoting lymphangiogenesis (Table 5 , Fig. 3f).

\section{Knockdown of DSG2 expression decreased CC cell proliferation and migration}

To determine the function of DSG2 in CC cell proliferation and migration, further investigation was performed using the CCK- 8 assay and migration assay.
First, qRT-PCR and western blotting revealed that DSG2 expression in $\mathrm{SiHa}$ and HeLa cells was higher than that in other cells and NCT (Fig. 4a). Therefore, SiHa and HeLa were chosen for further experiments. DSG2 expression was downregulated in $\mathrm{SiHa}$ and HeLa cell lines by transfection of siRNA393 and siRNA613. The efficiencies of interference were confirmed by qRT-PCR and western blotting (Additional file 1: Figure S2). The CCK-8 assay showed that knockdown of DSG2 expression decreased the cell proliferative capacity (Fig. 4b). Migration assays showed that knockdown of DSG2 expression decreased cell migration (Fig. 4c).

\section{Discussion}

Using survival analyses and TCGA data, our study provided a series of prognosis-related genes of early-stage CC. With GO, KEGG and PPI analyses, we can determine the main function distribution and interaction of genes. We identified 5 genes that were upregulated in early CC compared with normal samples that were correlated with unfavorable prognosis, including DSG2, matrix metallopeptidase 1 (MMP1), carbonic anhydrase IX (CA9), homeobox A1 (HOXA1), and serine protease inhibitor B3 (SERPINB3). MMP1, CA9, HOXA1 and SERPINB3 had been explored. However, we could not find any studies exploring the relationship between DSG2 and CC. To the best of our knowledge, this is the first one.

Consistent with the TCGA data mining result, DSG2 was more highly expressed in CC tissue than in normal tissue in 4 Oncomine databases. By detecting DSG2 expression in tissues, we revealed that DSG2 was upregulated in CC tissue compared with ANT or NCT. Additionally, DSG2 was significantly correlated with tumor size, PLNM, recurrence and vital status in 5 years, but not FIGO stage, pathologic type, differentiation grade, stromal invasion, LVSI, vaginal involvement and parametrial infiltration. High DSG2 expression predicted an unfavorable prognosis in early-stage CC. As PLNM was the most important risk factor for CC development, we investigated the relationship between DSG2 and PLNM by IHC, qRT-PCR and western blot analyses. 


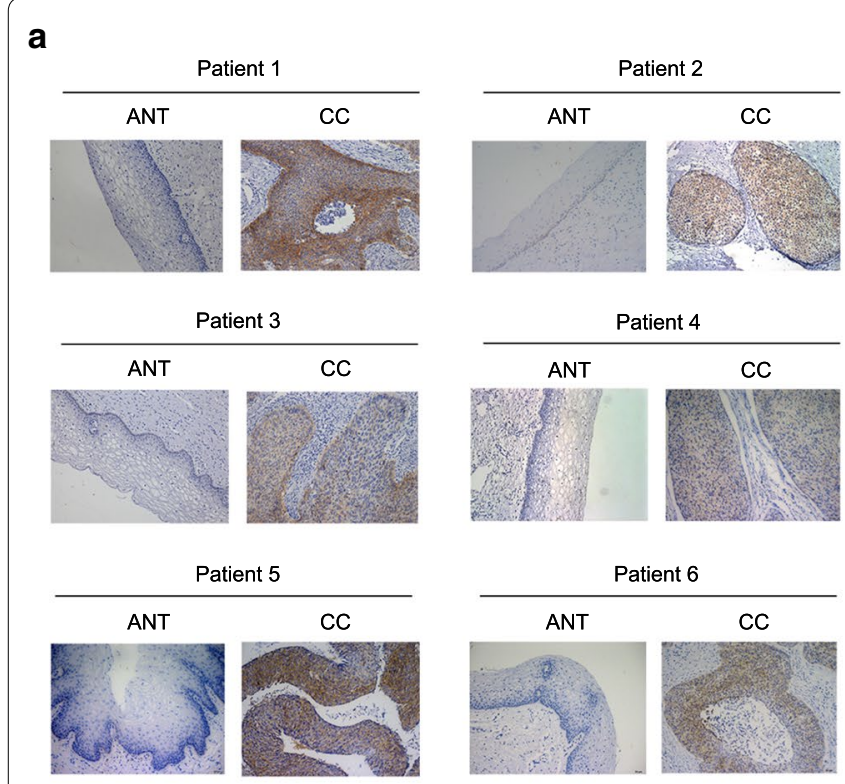

b
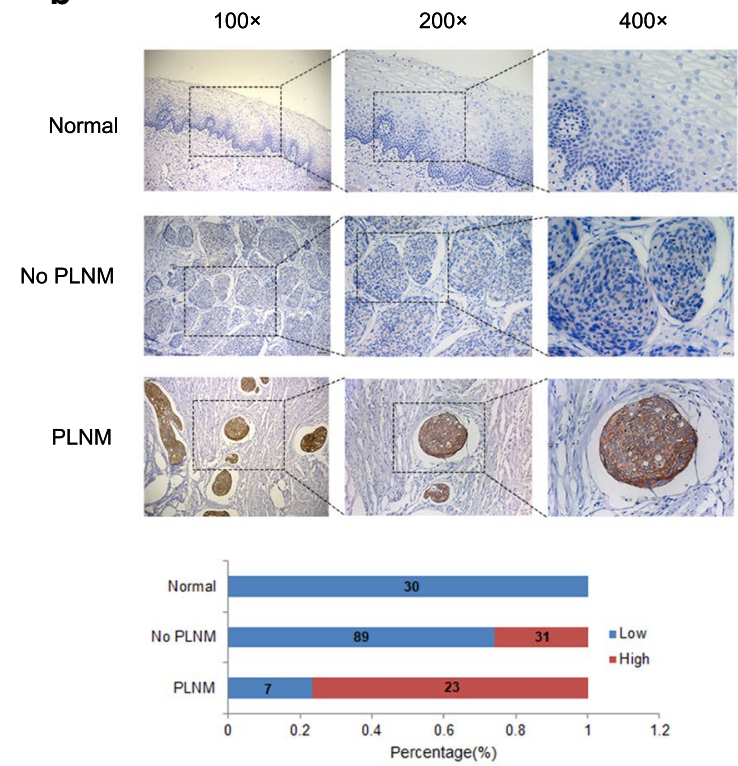

。

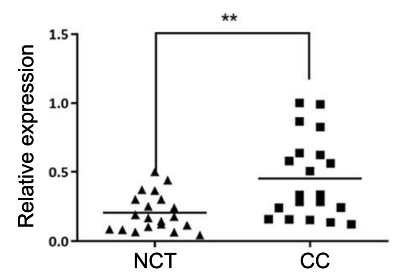

d

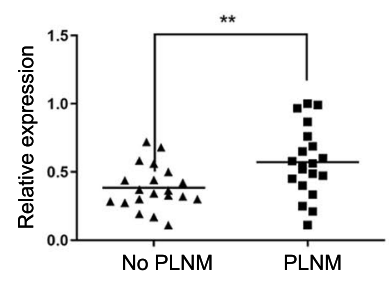

e

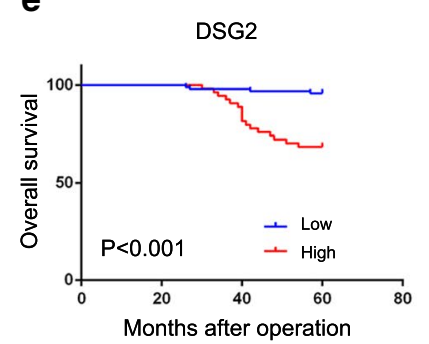

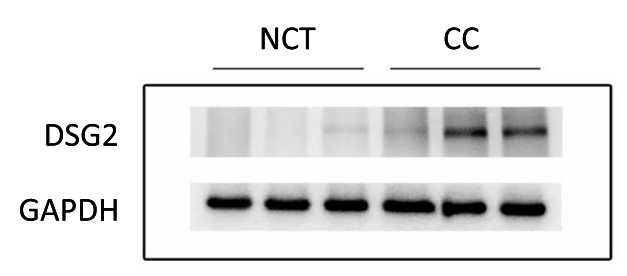
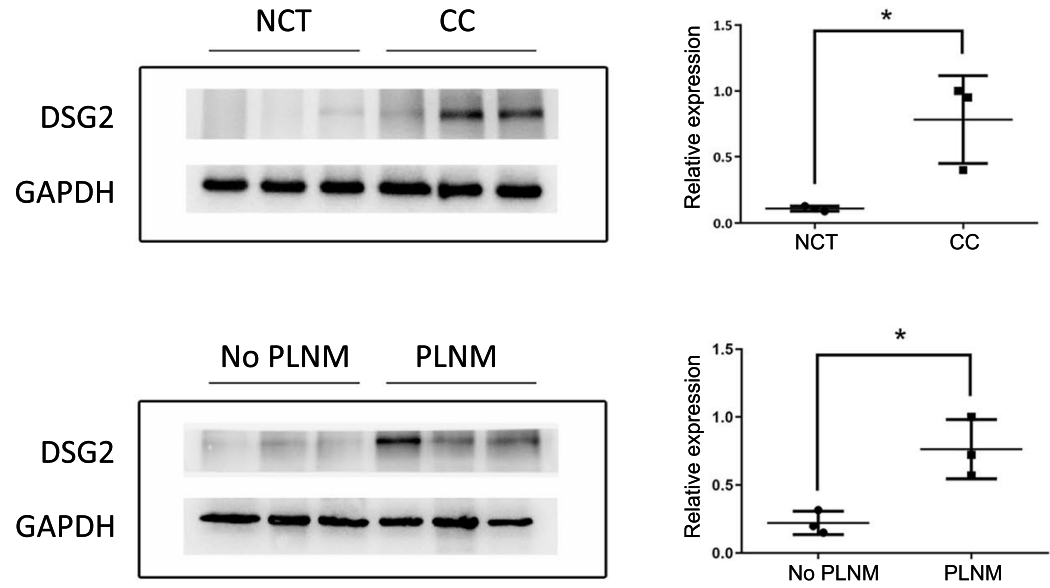

DSG2

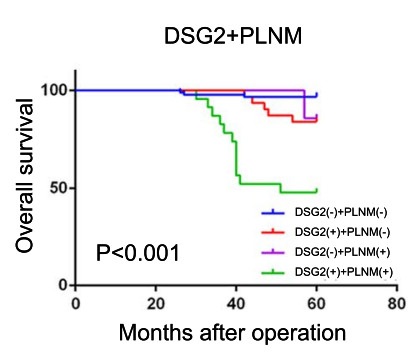

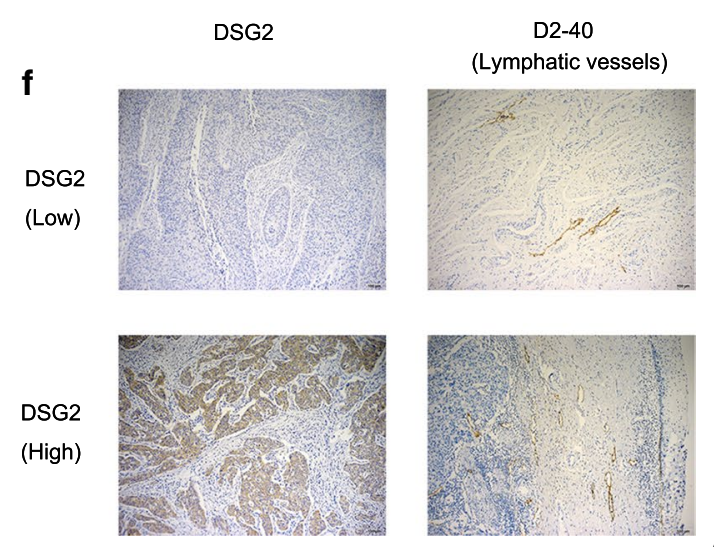


Table 2 Correlation between DSG2 expression and clinicopathological features of early-stage CC

Variables Total $\frac{\text { DSG2 expression }}{\text { Low High }} P^{\text {a }}$

Age (years)

$\leq 42$

$>42$

FIGO stage

।

$\|$

Tumor size $(\mathrm{cm})$

$$
\leq 4
$$$$
>4
$$

Pathologic types

Squamous cell carcinoma

Adenocarcinoma

Adenosquamous carcinoma

Differentiation grade

Well

Moderate

Poor

Stromal invasion

$<1 / 2$

$\geq 1 / 2$

Lymphovascular space invasion

No

Yes

Pelvic lymph node metastasis

$$
\text { No }
$$

Yes

Vaginal involvement

$$
\text { No }
$$

Yes

Parametrial infiltration

$$
\text { No }
$$$$
\text { Yes }
$$

Recurrence

$$
\text { No }
$$

Yes

Vital status in 5 years

\begin{tabular}{lrrrr} 
Alive & 129 & 92 & 37 & $<0.001$ \\
Dead & 21 & 4 & 17 & \\
\hline
\end{tabular}

a $P$ value from Fisher's exact test; The italic number inside the table reflected $P<0.05$

${ }^{b}$ FIGO 2009 was used

All experiments showed that DSG2 was more highly expressed in the PLNM group than in the non-PLNM group. Moreover, high DSG2 expression was associated with high LMVD. Furthermore, our in vitro studies demonstrated that knockdown of DSG2 inhibited the CC cell
Table 3 Univariate Cox analysis of factors associated with overall survival in early-stage cohort

\begin{tabular}{lll}
\hline Variables & HR $(\mathbf{9 5} \% \mathbf{C l})^{\mathbf{a}}$ & $\boldsymbol{P}$ \\
\hline Age (years) & $1.007(0.960-1.056)$ & 0.774 \\
$\begin{array}{l}\text { Tumor size (cm) } \\
\quad \leq 4 \text { (reference) }\end{array}$ & 1 & \\
$>4$ & $2.750(1.110-6.814)$ & 0.029 \\
FIGO stage & & \\
$\quad$ I (reference) & 1 & 0.739 \\
| & $0.812(0.239-2.757)$ &
\end{tabular}

Pathologic types

Squamous cell carcinoma (refer- 1 ence)

\begin{tabular}{lll} 
Adenocarcinoma & $2.605(0.946-7.171)$ & 0.064 \\
Adenosquamous carcinoma & $2.583(0.341-19.558)$ & 0.358 \\
Differentiation grade & & \\
Well (reference) & 1 & 0.94 \\
Moderate & $6009.923(-)$ & 0.935 \\
Poor & $12,458.308(-)$ & \\
Stromal invasion & & 0.406 \\
$<1 / 2$ (reference) & 1 & \\
$\geq 1 / 2$ & $0.681(0.275-1.687)$ & \\
Lymphovascular space invasion & & \\
No (reference) & 1 & \\
Yes & $3.622(1.403-9.349)$ & \\
Pelvic lymph node metastasis & & \\
No (reference) & 1 & \\
Yes & $8.297(3.428-20.078)$ & \\
Vaginal involvement & & 0.001 \\
No (reference) & 1 & \\
Yes & $0.049(0-483,831.748)$ & \\
Parametrial infiltration & & \\
No (reference) & 1 & \\
Yes & $0.049(0-368,184,919.781)$ & 0.795 \\
DSG2 & 1 & \\
Low (reference) & $8.679(2.917-25.822)$ & \\
High & & \\
\hline a & & \\
\hline
\end{tabular}

a $95 \% \mathrm{Cl}, 95 \%$ confidence interval; $\mathrm{HR}$, hazard ratio

b FIGO 2009 was used

proliferative capacity and migration ability. In conclusion, DSG2 was a novel tumor promoter in CC, and probably promoted cancer development by promoting the occurrence of PLNM.

However, some studies showed that the downregulation of DSG2 promoted the proliferation and metastasis of cancer cells because desmosome downregulation decreases adhesion junctions to drive tumor development and early invasion. The reasons why our results were contrary to some studies were probably as follows. First, as we found above, DSG2 played a different role in 
Table 4 Multivariate Cox analysis of factors associated with overall survival in early-stage cohort

\begin{tabular}{lll}
\hline Variables & HR $(\mathbf{9 5} \% \mathbf{C l})^{\mathbf{a}}$ & $P$ \\
\hline $\begin{array}{l}\text { Tumor size (cm) } \\
\leq 4 \text { (reference) }\end{array}$ & 1 & \\
$>4$ & $1.704(0.646-4.498)$ & 0.282 \\
$\begin{array}{l}\text { Lymphovascular space invasion } \\
\quad \text { No (reference) }\end{array}$ & 1 & \\
Yes & $2.595(0.946-7.123)$ & 0.064 \\
Pelvic lymph node metastasis & & \\
No (reference) & 1 & \\
$\quad$ Yes & $3.935(1.480-10.465)$ & 0.006 \\
DSG2 & & \\
Low (reference) & 1 & 0.018 \\
High & $4.234(1.275-14.063)$ & \\
\hline
\end{tabular}

a $95 \% \mathrm{Cl}, 95 \%$ confidence interval; $\mathrm{HR}$, hazard ratio

Table 5 Correlation between DSG2 expression and lymphatic vessel density of early-stage CC

\begin{tabular}{llll}
\hline & \multicolumn{2}{l}{ DSG2 expression } & P \\
\cline { 2 - 3 } & Low & High & \\
\hline Lymphatic vessel density & $6.2 \pm 3.4$ & $13.4 \pm 2.8$ & 0.034 \\
\hline
\end{tabular}

different kinds of cancer. Second, our results showed that high DSG2 expression was correlated with high LMVD, indicating that DSG2 may promote the occurrence of PLNM by promoting lymphangiogenesis. Cell adhesion was not an important factor in CC progression. Third, DSG2 may be a component of the metastasis pathway, regulating cell migration indirectly.

DSG2 plays a different role in different kinds of cancer. With TCGA data mining, we found that high DSG2 expression was correlated with the unfavorable prognosis of BLCA, brain LGG, LUAD, PAAD and UCEC, while high DSG2 expression was correlated with the favorable prognosis of COAD, KIRC and KIRP. These findings were consistent with those of previous reports. DSG2 is probably a novel biomarker of cancers but has different functions in different cancers.

Desmosomal cadherins are a component in cell-cell junctions, which are involved in the process of intercellular communication, signal transduction and cell proliferation [20]. In addition to regulating cell adhesion, DSG2 influenced cell proliferation and invasion by regulating the signaling pathway. It could be upstream or downstream of a pathway. The coexpression analyses results showed that cell division, positive regulation of transferase activity, positive regulation of cell migration and the EGFR upregulation pathway were significantly enriched among the positively correlated genes, revealing that DSG2 is involved in the process and metastasis of CC. These results were consistent with those of previous reports. Cai et al. [12] showed that knockdown of DSG2 suppressed non-small cell lung cancer cell proliferation by targeting p27 and CDK2. Kamekura et al. [10] reported that DSG2 and DSC2 played opposite roles in colon cancer cell proliferation. The loss of DSG2 suppressed cell proliferation through the altered phosphorylation of EGFR, Src and Erk protein. Overmiller et al. [21] suggested that in skin squamous cell carcinoma, DSG2 stimulated cell growth and migration by positively regulating EGFR levels and signaling through a c-Src and Cav1-dependent mechanism using lipid rafts as signal modulatory platforms. BrennanCrispi et al. [22] showed that in skin basal cell carcinoma and squamous cell carcinoma, DSG2 enhanced canonical hedgehog signaling downstream of Ptc1 to promote cancer development through the activation of phosphorylated Stat 3 and regulation of Gli1 expression. Katharina et al. [23] identified a novel promigratory pathway of pancreatic cancer cells in which the loss of DSG2 reduces the levels of plakoglobin via deregulated MAPK signaling. All of the above results showed that DSG2 was involved in various signaling pathways, such as the EGFR and MAPK signaling pathways as well as cell cycle pathways, indicating its important function in signaling pathway regulation.

Our study was the first to investigate the relationship between DSG2 expression and lymphangiogenesis. Coexpression analyses showed that DSG2 was positively correlated with CCBE1, which positively regulated lymphangiogenesis, while it was negatively correlated with VASH1, which negatively regulated lymphangiogenesis. An experiment was conducted to detect LMVD in tissue, which has not been reported in previous studies, and high LMVD was found to be associated with high DSG2 expression, indicating that DSG2 probably increased the lymphangiogenesis of cancer.

In conclusion, our current study was the first to show that DSG2 was overexpressed in CC tumorigenesis and that DSG2 knockdown repressed CC cell proliferation and migration. However, further mechanisms and signaling pathways underlying the role of DSG2 in CC remain to be defined.

\section{Conclusions}

Based on the above data, we drew a conclusion that DSG2 was a biomarker that promotes CC cells proliferation and metastasis and is correlated with poor prognosis in early-stage CC. These findings facilitated us to discover novel targets for the therapy of patients with CC. 


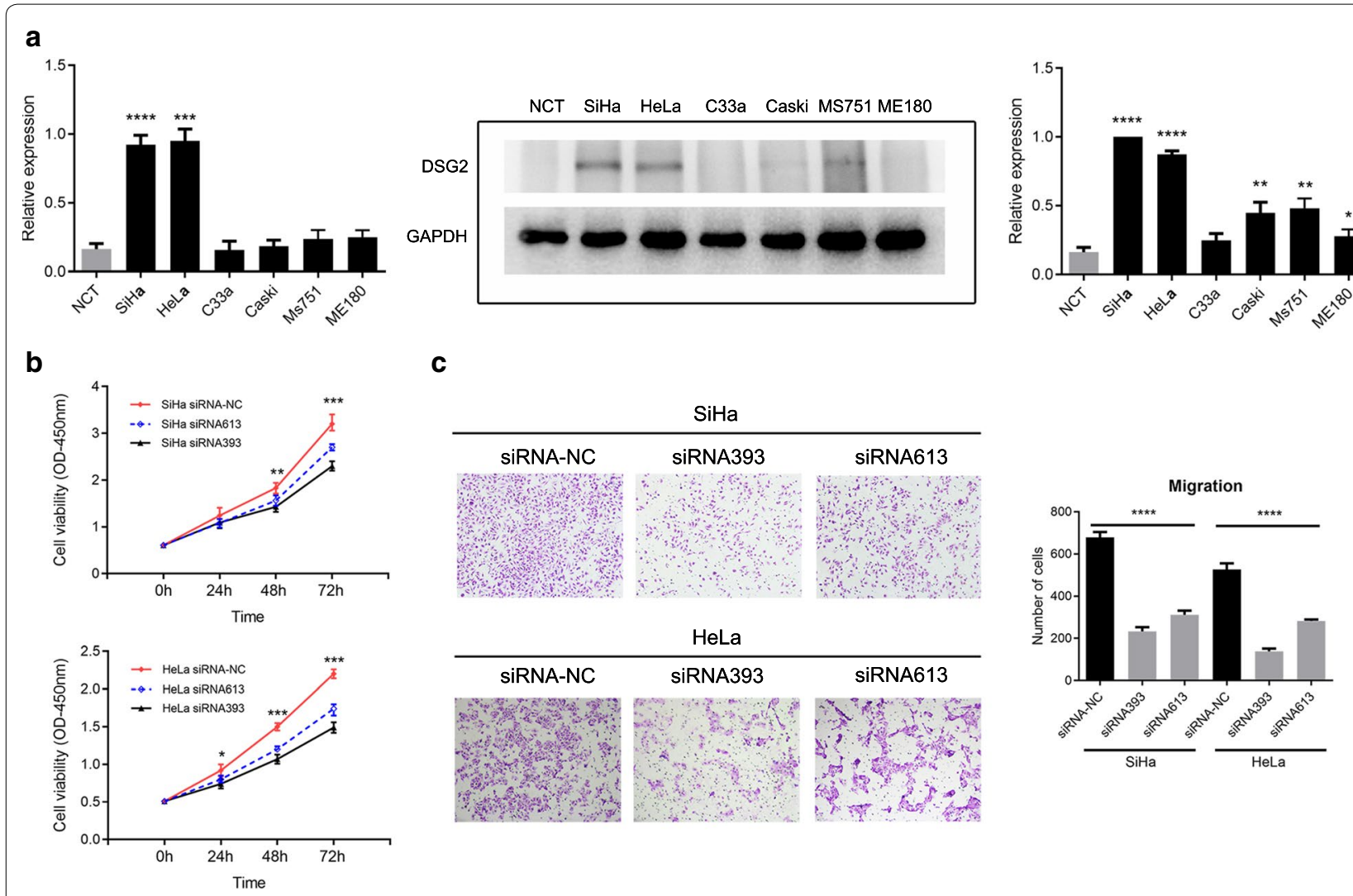

Fig. 4 The effect of DSG2 knockdown on the viability and migration of CC cells. a Analysis of the expression of DSG2 in six CC cell lines and NCTS by qRT-PCR (left) and western blotting (right). $\mathbf{b}$ The effect of siRNA on the viability of CC cells detected by CCK-8 assays. $\mathbf{c}$ The effect of siRNA on the migration abilities of CC cells detected by migration assay. Original magnification: $\times 100 .{ }^{*} P<0.05$; ${ }^{* *} P<0.01$; ${ }^{* * *} P<0.001$; ${ }^{* * *} P<0.0001$

\section{Supplementary information}

Supplementary information accompanies this paper at https://doi. org/10.1186/s12935-020-01292-x.

Additional file 1. Supplemental Figure S1-S2 : Figure S1. The bioinformatic analyses of prognosis-relative gene. a GO terms identified in the $\mathrm{GO}$ analysis for correlated coding genes in the cell component categories with 5 minimum $P$-adjusted values. $\mathrm{GO}$ terms identified in the $\mathrm{GO}$ analysis for correlated coding genes in the molecular function categories with 5 minimum Pvalues (All P-adjusted value $=1$ ). $\mathbf{b}$ Chromosome distribution of prognosis-relative gene. $\mathbf{c}$ Protein-protein interaction network of prognosis-relative gene. $\mathbf{d} \mathrm{GO}$ terms identified in the $\mathrm{GO}$ biological process analysis for negatively coexpressed genes in categories with 20 minimum P-adjusted values. Oncogenic signature for negatively coexpressed genes with 20 minimum P-adjusted values. The number of enriched oncogenic signatures for negatively correlated genes was only $\mathbf{1 8}$. Figure $\mathbf{S 2}$. The effect of siRNA on CC cells detected by qRT-PCR (a) and Western blot (b). ****P $<0.0001$

\section{Abbreviations}

CC: Cervical cancer; TCGA: The Cancer Genome Atlas; DSG2: Desmoglein-2; IHC: Immunohistochemistry; qRT-PCR: Quantitative real-time PCR; OS: Overall survival; CCK-8: Cell Counting Kit-8; MMP1: Matrix metallopeptidase 1; CA9: Carbonic anhydrase IX; HOXA1: Homeobox A1; SERPINB3: Serine protease inhibitor B3; ANT: Adjacent noncancerous tissue; PLNM: Pelvic lymph node metastasis; LVSI: Lymphovascular space invasion; NSCLC: Nonsmall cell lung cancer; KM: Kaplan-Meier; GO: Gene Ontology; KEGG: Kyoto
Encyclopedia of Genes and Genomes; PPI: Protein-protein interaction; FDR: False discovery rate; DEA: Differential expression analyses; NCT: Normal cervical tissue; BLCA: Bladder urothelial carcinoma; LGG: Brain lower-grade glioma; LUAD: Lung adenocarcinoma; PAAD: Pancreatic adenocarcinoma; UCEC: Uterine corpus endometrial carcinoma; COAD: Colon adenocarcinoma; KIRC: Kidney renal clear cell carcinoma; KIRP: Kidney renal papillary cell carcinoma; LMVD: Lymphatic microvessel density.

\section{Acknowledgements}

Not applicable.

\section{Authors' contributions}

$S Q, Y L, Q D, C S$ and SY contributed to the study conception and design. Material preparation and data collection were performed by SQ, WW, JH, TL and $M X$. The experiments were performed mainly by $S Q$, with help from $Y L$ and $P L$. Analysis was performed by $\mathrm{SQ}$. The first draft of the manuscript was written by SQ. All authors have confirmed the submission of this manuscript. All authors read and approved the final manuscript.

\section{Funding}

This study was supported by grants from National Natural Science Foundation of China (Nos. 81672561, 81874102) (S.Z. Yao); Sun Yat-Sen University Clinical Research 5010 Program (No. 2017006) (S.Z. Yao).

\section{Availability of data and materials}

The datasets used and analyzed during the current study are available from the corresponding author on reasonable request. 


\section{Ethics approval and consent to participate}

The study was approved by the local ethical committee of the First Affiliated Hospital of Sun Yat-sen University and all study subjects gave written informed consent.

\section{Consent for publication}

Not applicable.

\section{Competing interests}

The authors declare that they have no competing of interest.

\section{Author details}

${ }^{1}$ Department of Obstetrics and Gynecology, The First Affiliated Hospital, Sun Yat-sen University, Zhongshan Second Road 58, Guangzhou 510080, People's Republic of China. ${ }^{2}$ Department of Obstetrics and Gynecology, Peking University Third Hospital, Beijing 100191, People's Republic of China.

Received: 8 April 2020 Accepted: 25 May 2020

Published online: 03 June 2020

\section{References}

1. Bray F, et al. Global cancer statistics 2018: GLOBOCAN estimates of incidence and mortality worldwide for 36 cancers in 185 countries. CA Cancer J Clin. 2018:68(6):394-424.

2. Huang BX, Fang F. Progress in the Study of Lymph Node Metastasis in Early-stage Cervical Cancer. Curr Med Sci. 2018;38(4):567-74.

3. Kwon J, et al. The prognostic impact of the number of metastatic lymph nodes and a new prognostic scoring system for recurrence in early-stage cervical cancer with high risk factors: a Multicenter Cohort Study (KROG 15-04). Cancer Res Treat. 2018;50(3):964-74.

4. Cancer Genome Atlas Research Network. Integrated genomic and molecular characterization of cervical cancer. Nature. 2017;543(7645):378-84.

5. Tomczak K, Czerwińska P, Wiznerowicz M. The Cancer Genome Atlas (TCGA): an immeasurable source of knowledge. Contemp Oncol (Pozn). 2015;19(1a):A68-77.

6. Hermida A, et al. High risk of heart failure associated with desmoglein-2 mutations compared to plakophilin-2 mutations in arrhythmogenic right ventricular cardiomyopathy/dysplasia. Eur J Heart Fail. 2019;21(6):792-800

7. Zhou G, et al. The role of desmosomes in carcinogenesis. Onco Targets Ther. 2017;10:4059-63.

8. Brennan D, Mahoney MG. Increased expression of Dsg2 in malignant skin carcinomas: a tissue-microarray based study. Cell Adh Migr. 2009;3(2):148-54
9. Kurzen H, Münzing I, Hartschuh W. Expression of desmosomal proteins in squamous cell carcinomas of the skin. J Cutan Pathol. 2003;30(10):621-30.

10. Kamekura $\mathrm{R}$, et al. Loss of the desmosomal cadherin desmoglein-2 suppresses colon cancer cell proliferation through EGFR signaling. Oncogene. 2014;33(36):4531-6.

11. Saaber $F$, et al. Expression of desmogleins 1-3 and their clinical impacts on human lung cancer. Pathol Res Pract. 2015;211(3):208-13.

12. Cai $F$, et al. Desmoglein- 2 is overexpressed in non-small cell lung cancer tissues and its knockdown suppresses NSCLC growth by regulation of p27 and CDK2. J Cancer Res Clin Oncol. 2017;143(1):59-69.

13. Yashiro M, Nishioka N, Hirakawa K. Decreased expression of the adhesion molecule desmoglein-2 is associated with diffuse-type gastric carcinoma. Eur J Cancer. 2006:42(14):2397-403.

14. Ramani VC, Hennings $L$, Haun RS. Desmoglein 2 is a substrate of kallikrein 7 in pancreatic cancer. BMC Cancer. 2008;8:373.

15. Barber $A G$, et al. Characterization of desmoglein expression in the normal prostatic gland Desmoglein 2 is an independent prognostic factor for aggressive prostate cancer. PLOS ONE. 2014;9(6):98786.

16. Davies $\mathrm{E}_{\text {, et }} \mathrm{al}$. The role of desmoglein 2 and $\mathrm{E}$-cadherin in the invasion and motility of human breast cancer cells. Int J Oncol. 1997:11(2):415-9.

17. Sidaway P. Pancreatic cancer: TCGA data reveal a highly heterogeneous disease. Nat Rev Clin Oncol. 2017;14(11):648.

18. Zhu Y, Qiu P, Ji Y. TCGA-assembler: open-source software for retrieving and processing TCGA data. Nat Methods. 2014;11(6):599-600.

19. Rhodes DR, et al. Oncomine 30: genes, pathways, and networks in a collection of 18,000 cancer gene expression profiles. Neoplasia. 2007:9(2):166-80

20. Oren $\mathrm{O}, \mathrm{Smith} \mathrm{BD}$. Eliminating cancer stem cells by targeting embryonic signaling pathways. Stem Cell Rev. 2017;13(1):17-23.

21. Overmiller AM, et al. c-Src/Cav1-dependent activation of the EGFR by Dsg2. Oncotarget. 2016:7(25):37536-55.

22. Brennan-Crispi DM, et al. Overexpression of Desmoglein 2 in a mouse model of gorlin syndrome enhances spontaneous basal cell carcinoma formation through STAT3-Mediated Gli1 expression. J Invest Dermatol. 2019;139(2):300-7.

23. Hutz K, et al. Loss of desmoglein 2 promotes tumorigenic behavior in pancreatic cancer cells. Mol Carcinog. 2017;56(8):1884-95.

\section{Publisher's Note}

Springer Nature remains neutral with regard to jurisdictional claims in published maps and institutional affiliations.
Ready to submit your research? Choose BMC and benefit from

- fast, convenient online submission

- thorough peer review by experienced researchers in your field

- rapid publication on acceptance

- support for research data, including large and complex data types

- gold Open Access which fosters wider collaboration and increased citations

- maximum visibility for your research: over 100M website views per year

At BMC, research is always in progress.

Learn more biomedcentral.com/submissions 